\title{
Quasi-Chemical Approach to the Thermodynamics of Dilute Solution of an Interstitial Solute in a Multi-Component Substitutional Solvent
}

\author{
By M. L. Kapoor*
}

\begin{abstract}
Semigrand partition function of an assembly of $m$ substitutional and an interstitial component is constructed. This is evaluated on the basis of simplified assumptions of quasi-chemical theory. The resulting expression obtained for the solubility of interstitial component in a multi-component substitutional solvent are found to explain satisfactorily the behaviour of nitrogen in ternary substitutional alloys.
\end{abstract}

(Received June 17, 1976)

\section{Introduction}

In recent years considerable theoretical work has been done on the prediction and explanation of thermodynamic properties of interstitial solutes such as carbon, nitrogen and oxygen in binary substitutional alloys with the help of the thermodynamic properties of these solutes in corresponding metals. Though for practical purposes one requires the thermodynamic data of these solutes in multi-component systems, little has been done to extend the existing theories on the behaviour of these solutes in binary systems to aforementioned systems. The aim of the present work, therefore, is to develop a quasi-chemical approach for the calculation of thermodynamic properties of dilute interstitial solute in multi-component molten substitutional solvents.

\section{Theoretical}

Let us consider an $m$-component substitutional solvent, containing $n_{1}, n_{2}, \ldots, n_{m}$ atoms of components $1,2, \ldots, m$ respectively, in contact with another surrounding phase having a chemical potential $\mu_{x}$ for a component $x$ which dissolves in the aforementioned solvent as an interstitial component. Semi-grand partition function of the solution will be

* Department of Metallurgical Engineering, University of Roorkee, Roorkee (U.P.), India.

$$
\Xi=\sum_{n_{x}} \sum_{r} \Omega\left(n_{1}, n_{2}, \ldots, n_{m}, n_{x}\right) \exp \left(\frac{n_{x} \mu_{x}}{N k T}\right)
$$

where $r$ denotes all distinguishable accessible states of the system, $\Omega\left(n_{1}, n_{2}, \ldots, n_{m}, n_{x}\right)$ represents the configurational partition function of the solution when it contains $n_{x}$ solute atoms, $N$ is Avogadro's number, $k$ is Boltzmann's constant, and $T$ is the absolute temperature. To evaluate the right hand side of eq. (1) let us make the following simplying assumptions:

(1) The solution forms a three dimensional lattice in which solvent atoms occupy the substitutional and the solute atoms the interstitial sites. Each site is occupied by at the most one atom and there are no substitutional vacancies in the system. Thus the total number of interstitial sites $\left(n_{\text {int }}\right)$ and interstitial vacancies $\left(n_{v}\right)$ in the system will be

$$
\begin{aligned}
n_{\mathrm{int}} & =\rho_{x} n_{s} \\
n_{v} & =\left(\rho_{x} n_{s}-n_{x}\right)
\end{aligned}
$$

where $\rho_{x}$ is the number of interstitial sites per substitutional site, and $n_{s}$ is the total number of substitutional sites in the system and is equal to the sum of all types of substitutional atoms.

(2) Each solute atom possesses the same coordination number $z_{x}$ with the substitutional atoms. Thus a solute atom having $i_{1}, i_{2}, \ldots$, $i_{m}$ atoms of components $1,2, \ldots, m$ respectively, in its coordination shell can assume ${ }^{z_{x}} C_{i_{1}} \cdot\left(z_{x}-i_{1}\right) C_{i_{2}} \ldots\left(z_{x}-i_{1} \cdots i_{m-1}\right) C_{i_{m-1}}$ distinguishable configurations. 
(3) Energy of a solute atom is assumed to be equal to the sum of the energies contributed by the pairs formed by the solute atom with its substitutional neighbours. The energy contribution to an $x$ atom of a pair is assumed to be fixed when the substitutional partner of the pair is named. This assumption is similar to the one made by Fowler and Guggenheim ${ }^{(1)}$ in their treatment of strictly regular solutions. Accordingly, the energy $\left(W_{x}^{1_{1}, 2_{2} \cdots 1_{m}}\right)$ of an $x$ atom surrounded by $l_{1}, l_{2}, \ldots, l_{m}$ atoms of components $1,2, \ldots, m$ respectively will be

$$
W_{x}^{l_{1} \cdots l_{m}}=\sum_{i} \frac{l_{i}}{z_{x}} W_{x}^{i}
$$

where $W_{x}^{i}$ is the energy of an $x$ atom when it is surrounded by $z_{x}$ atoms of component $i$. Similarly one can write for the energy $\left(W_{k}^{j_{1}, j_{2}, \cdots, j_{m}, 1}\right)$ of an atom of component $k$ surrounded by $j_{1}, j_{2}, \ldots, j_{m}$ atoms of substitutional components $1,2, \ldots, m$ respectively and one atom of component $x$, the expression

$$
W_{k}^{j_{1}, j_{2}, \cdots, j_{m}, 1}=W_{k}^{j_{1}, \cdots j_{m}, 0}+W_{k}^{1}
$$

where $W_{k}^{1}$ is the energy contribution of an $x$ atom when it is occupying a neighbouring interstitial site of an atom of component $k$ surrounded by the atoms of its own type only at substitutional positions.

(4) Similar linear forms as in (3) are assumed to hold good for the energy contribution due to changes in the internal partition functions of various atoms. Thus

$$
\begin{aligned}
\ln q_{x}^{l_{1}, \cdots, l_{m}} & =\sum_{i} \frac{l_{i}}{z_{x}} \ln q_{x}^{i} \\
\ln q_{k}^{j_{1}, j_{2}, \cdots j_{m}, 1} & =\ln q_{k}^{j_{1}, j_{2}, \cdots, j_{k}}+\ln q_{k}^{1}
\end{aligned}
$$

(5) The summation in eq. (1) can be re- placed by the maximum term in the series when it is expanded in such a way that the terms for the same energy are written together ${ }^{(2)}$. Thus,

$$
\Xi=g\left(E_{\max }\right) \exp \left(-\frac{E_{\max }}{N k T}\right) \exp \left(\frac{n_{x}^{*} \mu_{x}}{N k T}\right) .
$$

(6) The number of atoms of component $x$ in the system is so small that no two neighbouring interstitial sites are simultaneously occupied by it and no substitutional atom has more than one interstitial atom at the interstitial sites surrounding it.

(7) Exchange of atoms of component $x$ by the system with its surroundings does not cause a rearrangement of solvent atoms at substitutional positions. This along with assumptions (3) and (4) allows one to split the right hand side of eq. (8) into two parts, namely, a substitutional part independent of component $x$ and an interstitial part dependent on component $x$. Thus, one can write eq. (8) in the form

$$
\begin{aligned}
\Xi= & {\left[g_{\text {sub }} \exp \left(-\frac{E_{\text {sub }}}{N k T}\right)\right] } \\
& \times\left[g_{\text {int }} \exp \left(-\frac{E_{\text {int }}}{N k T}\right) \exp \left(\frac{n_{x}^{*} \mu_{x}}{N k T}\right)\right] .
\end{aligned}
$$

where $g_{\text {sub }}$ and $g_{\text {int }}$ are the combinational terms due to the arrangements of substitutional and interstitial components respectively, $E_{\text {int }}$ and $E_{\text {sub }}$ are the energy contribution to the total energy $E_{\max }$ of the system due only to interstitial and substitutional components respectively, and $n_{x}$ is the number of atoms of component $x$ corresponding to the maximum term as denoted by eq. (8).

Following Pascal et al. ${ }^{(3)}$ one can write the following expression for $g_{\text {int }}$ :

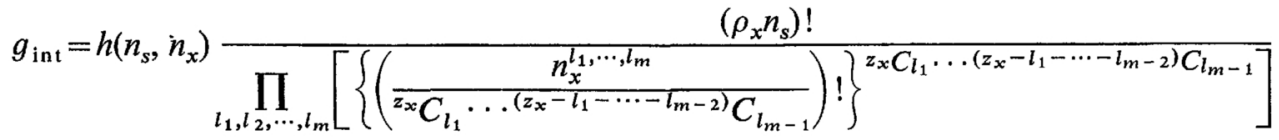

$$
\begin{aligned}
& \times \frac{1}{\prod_{p_{1}, p_{2}, \cdots, p_{m}}\left[\left\{\left(\frac{n_{v}^{p_{1}, p_{2}, \cdots, p_{m}}}{z_{x} C_{p_{1}} \cdots\left(z_{x}-p_{1}-\cdots-p_{x-2)} C_{p_{m-1}}\right.}\right) !\right\}^{{ }_{x} C_{p_{1}} \cdots\left(z_{x}-p_{1}-\cdots-p_{m-2}\right) C_{p_{m}-1}}\right]}
\end{aligned}
$$

where $n_{x}^{l_{1}, \cdots, l_{m}}$ is the number of $x$-atoms having $l_{1}, l_{2}, \ldots, l_{m}$ atoms of components $1,2, \ldots, m$ respectively around it, $n_{v}^{p_{1}}, \ldots, p_{m}$ is the number of interstitial vacancies having $p_{1}, p_{2}, \ldots, p_{m}$ atoms of components $1,2, \ldots, m$ respectively around it and $h\left(n_{s}, n_{x}\right)$ is a normalising factor and is given by the expression: 


$$
\begin{aligned}
\sum g_{\mathrm{int}}= & \frac{\left(\rho_{x} n_{s}\right) !}{n_{x} ! n_{v} !}=\sum \frac{\left(\rho_{x} n_{s}\right) !}{\prod_{l_{1}, \cdots, l_{m}}\left[\left\{\left(\frac{n_{x}^{1 l_{1} \cdots l_{m}}}{z_{x} C_{l_{1}} \cdots\left(z_{x}-l_{1}-\cdots-l_{m-2}\right) C_{l_{m-1}}}\right) !\right\}^{z_{x} C_{l_{1}} \cdots\left(z_{x}-l_{1}-\cdots-l_{m-2}\right) C_{l_{m-1}}}\right]} \\
& \times \frac{h\left(n_{s}, n_{x}\right)}{\prod_{p_{1}, \cdots, p_{m}}\left[\left\{\left(\frac{n_{v}^{1 p_{1} \cdots p_{m}}}{z_{x} C_{p_{1}} \cdots\left(z_{x}-p_{1}-\cdots-p_{m-2}\right)} C_{p_{m-1}}\right) !\right\}^{z_{x} C_{p_{1}} \cdots\left(z_{x}-p_{1}-\cdots-p_{m}-2\right) C_{p_{m-1}}}\right]}
\end{aligned}
$$

where summation in the first term of the above equation represents all possible arrangements of $x$-atoms at the interstitial sites. The total number of these arrangements are given by the second term in the above equation. Summation in the third term is over all possible values of $n_{x}^{1 l_{1}, l_{2}, \cdots, l_{m}}$ and $n_{v}^{1 p_{1}, p_{2}, \cdots, p_{m}}$ in the system. Replacing both these summations by the maximum term in their series one can write the expression:

$$
\begin{aligned}
& g_{\mathrm{int}}^{\max }=\frac{\left(\rho_{x} n_{s}\right) !}{n_{x} ! n_{v} !}
\end{aligned}
$$

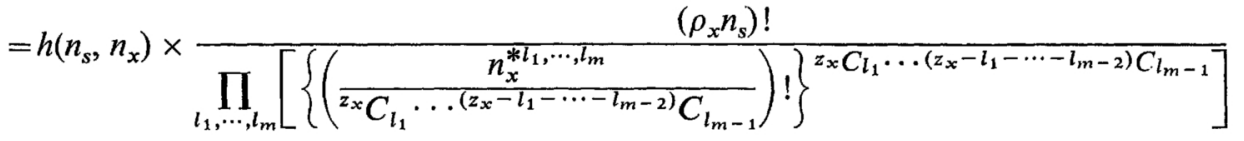

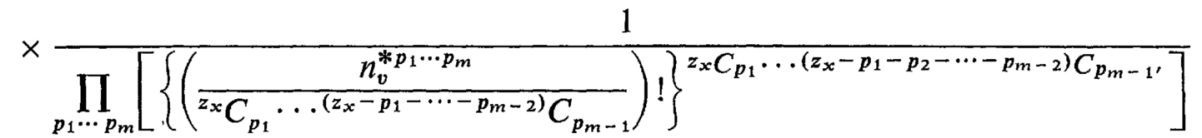

As $n_{x}^{* l_{1}, \cdots, l_{m}}$ and $n_{v}^{* p_{1}, \cdots, p_{m}}$ represent the number of $x$-atoms and vacancies respectively in the maximum term of the series in eq. (11), so these are determined by the conditions defined by the following expressions:

$$
\begin{array}{ll}
\frac{\partial \ln g_{\text {int }}}{\partial n_{x}^{* l_{1} \cdots l_{m}}}=0 & \text { for all } l_{1}, l_{2}, \ldots, l_{m} \text { sets } \\
\frac{\partial \ln g_{\text {int }}}{\partial n_{v}^{* p_{1} \cdots p_{m}}}=0 & \text { for all } p_{1}, p_{2}, \ldots, p_{m} \text { sets }
\end{array}
$$

Further $n_{x}^{* l_{1}, \cdots, l_{m}}$ and $n_{v}^{* p_{1}, \cdots, p_{m}}$ are subject to the following material balance constraints:

$$
\begin{aligned}
& \sum_{l_{1} \cdots l_{m}} n_{x}^{* l_{1} \cdots l_{m}}-n_{x}=0 \\
& \sum_{p_{1} \cdots p_{m}} n_{v}^{* p_{1} \cdots p_{m}}-\left(\rho n_{s}-n_{x}\right)=0 \\
& \sum_{l_{1} \cdots l_{m}} l_{1} n_{x}^{* l_{1} \cdots l_{m}}+\sum_{p_{1} \cdots p_{m}} p_{1} n_{v}^{* p_{1} \cdots p_{m}}-z_{s x} n_{1}=0 \\
& \vdots \\
& \sum_{l_{1} \cdots l_{m}} l_{m-1} n_{x}^{* l_{1} \cdots l_{m}}+\sum_{p_{1} \cdots p_{m}} p_{m-1} n_{v}^{* p_{1} \cdots p_{m}}-z_{s x} n_{m-1}=0
\end{aligned}
$$

where $z_{s x}$ is the number of interstitial sites around a substitutional one and is given by

$$
z_{s x}=\rho_{x} z_{x} .
$$

Substitution of eq. (12) in eqs. (13) and (14) and the combination of the resulting expressions with eqs. (15)-(17) by the lagrangian multiplier method leads to

$$
\begin{aligned}
& n_{x}^{* l_{1} \cdots l_{m}}={ }^{z_{x}} C_{l_{1}} \cdots\left(z_{x}-l_{1}-\cdots-l_{m-2)} C_{m-1} \exp \left(\alpha_{x}^{*}+\sum_{i=1}^{m-1} l_{i} \beta_{i}^{*}\right)\right. \\
& n_{v}^{* p_{1} \cdots p_{m}}={ }^{z_{x}} C_{p_{1}} \ldots{ }^{\left(z_{x}-p_{1}-\cdots-p_{m-2)}\right.} C_{p_{m-1}} \exp \left(\alpha_{v}^{*}+\sum_{i=1}^{m-1} p_{i} \beta_{i}^{*}\right)
\end{aligned}
$$


where $\alpha_{x}^{*}, \alpha_{v}^{*}$ and $\beta_{1}^{*}, \ldots, \beta_{i}^{*}, \ldots, \beta_{m-1}^{*}$ are the lagrangian multipliers for eqs. (15), (16) and $(17 \cdot 1) \ldots(17 \cdot \mathrm{m}-1)$ respectively and are determined by the substitution of eqs. (19) and (20) in eqs. (15)-(17): Thus, with eqs. (15), (16), (19) and (20) one gets

$$
\begin{aligned}
\sum_{l_{1} \cdots l_{m}} n_{x}^{* l_{1} \cdots l_{m}} & =\left(\exp \alpha_{x}^{*}\right)\left[1+\sum_{i=1}^{m-1} \exp \beta_{i}^{*}\right]^{z_{x}}=n_{x}, \\
\sum_{p_{1} \cdots p_{m}} n_{v}^{* p_{1} \cdots p_{m}} & =\left(\exp \alpha_{v}^{*}\right)\left[1+\sum_{i=1}^{m-1} \exp \beta_{i}^{*}\right]^{z_{x}}=\left(\rho_{x} n_{s}-n_{x}\right) .
\end{aligned}
$$

Let us now consider the maximisation of the function with respect to $n_{x}, n_{x}^{l_{1}, \cdots, l_{m}}, n_{v}^{p_{1}, \cdots, p_{m}}$. For this purpose, an expression for $E_{\text {int }} \times q_{\text {int }}$ is required apart from the expression for $g_{\text {int }}$ which is given by eq. (10). According to assumptions (3) and (4), one can write the following expression for $E_{\text {int }}$ (including $q_{\text {int }}$ ):

$$
\begin{aligned}
E_{\mathrm{int}}= & \sum_{l_{1} \cdots l_{m}}\left(E_{x}^{l_{1} \cdots l_{m}}-k T \ln q_{x}^{l_{1} \cdots l_{m}}\right) n_{x}^{l_{1} \cdots l_{m}} \\
& +\sum_{j_{1} \cdots j_{m}} \sum_{i}\left[\left(E_{i}^{j_{1} \cdots j_{m}, 1}-E_{i}^{j_{1} \cdots j_{m}, 0}\right)-k T\left(\ln q_{i}^{j_{1} \cdots j_{m}, 1}-\ln q_{i}^{j_{1} \cdots j_{m}, 0}\right)\right] n_{i}^{j_{1}, \cdots, j_{m}, 1} \\
= & \sum_{l_{1} \cdots l_{m}} \frac{l_{i}}{z}\left(W_{x}^{i}-k T \ln q_{x}^{i}\right) n_{x}^{l_{1} \cdots l_{m}}+\sum_{l_{1} \cdots l_{m}} l_{i}\left(W_{i}^{1}-k T \ln q_{i}^{1}\right) n_{i}^{l_{1} \cdots l_{m}} .
\end{aligned}
$$

The maximisation conditions for eq. (9) are:

$$
\begin{gathered}
\frac{\partial \ln \Xi}{\partial n_{x}}=0, \\
\frac{\partial \ln \Xi}{\partial n_{x}^{l_{1} \cdots l_{m}}}=0, \\
\frac{\partial \ln \Xi}{\partial n_{v}^{p_{1} \cdots p_{m}}}=0 .
\end{gathered}
$$

Maximisation is to be done under the following mass balance constraints:

$$
\begin{aligned}
& \sum_{l_{1} \cdots l_{m}} n_{x}^{l_{1} \cdots l_{m}}-n_{x}=0, \\
& \sum_{p_{1} \cdots p_{m}} n_{v}^{p_{1} \cdots p_{m}}-n_{v}=0, \\
& \sum_{l_{1} \cdots l_{m}} l, n_{x}^{l_{1} \cdots l_{m}}+\sum_{p_{1} \cdots p_{m}} p_{1} n_{v}^{p_{1} \cdots p_{m}}-z_{s x} n_{1}=0, \\
& \sum_{l_{1} \cdots l_{m}} l_{m-1} n_{x}^{l_{1} \cdots l_{m}}+\sum_{p_{1} \cdots p_{m}} p_{m-1} n_{v}^{p_{1} \cdots p_{m}}-z_{s x} n_{m-1}=0 .
\end{aligned}
$$

Substitution of eq. (10) and eq. (23) in eq. (9) and the application of the lagrangian multiplier method for the maximisation of the resulting expression under the constraints given by eqs. (27)-(29) and with the help of (12) and (15)-(17), one gets

$$
\begin{gathered}
\frac{n_{x}^{*}}{\rho_{x} n_{s}-n_{x}^{*}}=\exp \left[+\alpha_{x}^{*}-\alpha_{x}-\alpha_{v}^{*}+\alpha_{v}\right] \exp \left(\frac{b_{x}}{N k T}\right), \\
n_{x}^{l_{1} \cdots l_{m}}={ }^{z_{x}} C_{l_{1}} \cdots\left(z_{x}-l_{1}-\cdots-l_{m-2)} C_{l_{m-1}} \times \exp \alpha_{x}\right. \\
\times \exp \left[\sum_{i=1}^{m-1} l_{i}\left(\beta_{i}-\frac{W_{x}^{i}-k T \ln q_{x}^{i}+z_{x} W_{i}^{1}-z_{x} k T \ln q_{i}^{1}}{z_{x} k T}\right]\right. \\
\quad \times \exp \left[\frac{l_{m}}{z_{x} k T}\left(k T \ln q_{x}^{m}-W_{x}^{m}+z_{x} k T \ln q_{m}^{1}-z_{x} W_{m}^{1}\right)\right], \\
n_{v}^{p_{1} \cdots p_{m}}={ }^{z_{x}} C_{p_{1}} \cdots\left(z_{x}-p_{1}-\cdots-p_{m-2)} C_{p_{m-1}} \times \exp \alpha_{v} \times \exp \left(\sum l_{i} \beta_{i}\right) .\right.
\end{gathered}
$$


Here $\alpha_{x}, \alpha_{v}$ and $\beta_{1}, \ldots, \beta_{m-1}$ are the lagrangian multipliers for the eqs. (27), (28) and (29.1) ... $(29 \cdot \mathrm{m}-1)$ respectively. Summation of eqs. (31) and (32) over all configurations of the surrounding substitutional atoms leads to the expressions:

$$
\begin{aligned}
n_{x}^{*}=\sum_{l_{1} \cdots l m} n_{x}^{l_{1} \cdots l_{m}}= & \left(\exp \alpha_{x}\right)\left[\sum_{i=1}^{m-1} \exp \left(\beta_{i}-\frac{W_{x}^{i}-k T \ln q_{x}^{i}+z_{x} W_{x}^{i}-z_{x} k T \ln q_{x}^{1}}{z_{x} k T}\right)\right. \\
& \left.+\exp \left(-\frac{W_{x}^{m}-k T \ln q_{x}^{m}+z_{x} W_{m}^{1}-z_{x} k T \ln q_{m}^{1}}{z_{x} k T}\right)\right]^{z_{x}}, \\
n_{v}^{*} & =\sum_{p_{1} \cdots p_{m}} n_{v}^{p_{1} \cdots p_{m}}=\left(\exp \alpha_{v}\right) \times\left[1+\sum_{i=1}^{m-1} \exp \beta_{i}\right]^{z_{x}} .
\end{aligned}
$$

In order to calculate the thermodynamic properties of the interstitial component from its properties in pure liquid metallic solvent, the right hand side of eq. (30) should be expressed in terms of the parameters which can be determined from the thermodynamics of the interstitial component in pure solvent. For this purpose let us first eliminate $\alpha_{x}, \alpha_{v}, \alpha_{x}^{*}$ and $\alpha_{v}^{*}$ from eq. (30) with the help of eqs. (21), (22), (33) and (34). Thus one gets

$$
\begin{aligned}
\frac{n_{x}^{*}}{\rho_{x} n_{s}-n_{x}^{*}}= & \frac{\exp \left(\mu_{x} / N k T\right)}{\left(1+\sum_{i} \exp \beta_{i}\right)^{z_{x}}} \times\left[\exp \left\{-\left(\frac{W_{x}^{m}-k T \ln q_{x}^{m}+z_{x} W_{m}^{1}-z_{x} k T \ln q_{m}^{1}}{z_{x} k T}\right)\right\}\right. \\
& \left.+\sum_{i=1}^{m-1} \exp \left(\beta_{i}-\frac{W_{x}^{i}-k T \ln q_{x}^{i}+z_{x} W_{i}^{1}-z_{x} k T \ln q_{i}^{1}}{z_{x} k T}\right)\right]^{z_{x}} .
\end{aligned}
$$

Substitution of eq. (31) and eq. (32) in eq. $(29 \cdot 1)$ leads to the following expression with the help of eqs. (18), (33) and (34).

$$
\begin{aligned}
& \rho_{x} n_{1}=n_{v} \frac{\exp \beta_{1}}{1+\sum_{i=1}^{m-1} \exp \beta_{i}}+ \\
& \frac{n_{x}\left[\exp \left(\beta_{1}-\frac{W_{x}^{1}-k T \ln q_{x}^{m}}{z_{x} k T}-\frac{W_{1}^{1}-k T \ln q_{1}^{1}}{k T}\right)\right]}{\left[\sum_{i=1}^{m-1} \exp \left(\beta_{i}-\frac{W_{x}^{i}-k T \ln q_{x}^{i}}{z_{x} k T}-\frac{W_{i}^{1}-k T \ln q_{i}^{1}}{k T}+\exp \left(-\frac{W_{x}^{m}-k T \ln q_{x}^{m}+z_{x} W_{m}^{1}-z_{x} k T \ln q_{m}^{1}}{z_{x} k T}\right)\right]\right.} .
\end{aligned}
$$

As $n_{x} \ll n_{v}$ : one can assume $n_{v} \simeq n_{s}$ and neglect the term on the right hand side of the above equation. Thus,

$$
\frac{\exp \beta_{1}}{n_{1}}=\frac{1+\sum_{i=1}^{m-1} \exp \beta_{i}}{n_{s}}
$$

Proceeding in a similar way, one can arrive at the expression

$$
\frac{\exp \beta_{1}}{n_{1}}=\frac{\exp \beta_{2}}{n_{2}}=\cdots=\frac{\exp \beta_{m-1}}{n_{m-1}}=\frac{1}{n_{m}}=\frac{1+\sum_{i=1}^{m-1} \exp \beta_{i}}{n_{s}} .
$$

With the help of eqs. (31) and (33) one can write the expression

$$
\begin{aligned}
& \sum_{l_{1} \cdots l_{m}} l_{1} n_{x}^{l_{1} \cdots l_{m}} \\
& =\frac{z_{x} n_{x}\left[\exp \left(\beta_{1}-\frac{W_{x}^{1}-k T \ln q_{x}^{1}}{z_{x} k T}-\frac{W_{1}^{1}-k T \ln q_{1}^{1}}{k T}\right)\right]}{\left[\sum_{i=1}^{m-1} \exp \left(\beta_{i}-\frac{W_{x}^{i}-k T \ln q_{x}^{i}}{z_{x} k T}-\frac{W_{i}^{1}-k T \ln q_{i}^{1}}{k T}+\exp \left(-\frac{W_{x}^{m}-k T \ln q_{x}^{m}+z_{x} W_{m}^{1}-z_{x} k T \ln q_{m}^{1}}{z_{x} k T}\right)\right]\right.} .
\end{aligned}
$$


Substitution of the expression for $\exp \beta_{1}$ in the numerator of the right hand side of the above equation from eq. (37) and rearrangement of the various terms leads to the expression:

$$
\begin{gathered}
\frac{n_{s} \sum_{l_{1}, \cdots, l_{m}} l_{1} n_{x}^{l_{1}, \cdots, l_{m}}}{n_{1} \exp \left(-\frac{W_{x}^{1}-k T \ln q_{x}^{1}}{z_{x} k T}\right) \cdot \exp \left(-\frac{W_{1}^{1}-k T \ln q_{1}^{1}}{k T}\right)} \\
=\frac{z_{x} n_{x}}{\sum_{i=1}^{m-1}\left[\exp \left(\beta_{i}-\frac{W_{x}^{i}-k T \ln q_{x}^{i}+z_{x} W_{i}^{1}-z_{x} k T \ln q_{i}^{1}}{z_{x} k T}\right)\right]} \\
\times \frac{\left(1+\sum_{i=1}^{m-1} \exp \beta_{i}\right)}{\exp \left(-\frac{W_{x}^{m}-k T \ln q_{x}^{m}+z_{x} W_{m}^{1}-z_{x} k T \ln q_{1}^{1}}{z_{x} k T}\right)} .
\end{gathered}
$$

One can arrive at similar expressions for the number of atoms of other components surrounding component $x$ and thus write the expression:

$$
\begin{aligned}
& \frac{n_{s} \sum_{l_{1}, \cdots, l_{m}} l_{1} n_{x}^{l_{1}, \cdots, l_{m}}}{n_{1} \exp \left(-\frac{W_{x}^{1}-k T \ln q_{x}^{1}}{z_{x} k T}\right) \cdot \exp \left(-\frac{W_{1}^{1}-k T \ln q_{1}^{1}}{k T}\right)} \\
&= \ldots \\
&= \frac{n_{s} \sum_{l_{1} \cdots l_{m}} l_{m} n_{x}^{l_{1} \cdots l_{m}}}{n_{m} \exp \left(-\frac{W_{x}^{m}-k T \ln q_{x}^{m}}{z_{x} k T}\right) \cdot \exp \left(-\frac{W_{m}^{1}-k T \ln q_{m}^{1}}{k T}\right)} \\
&= \frac{n_{s} \sum_{i=1}^{m} \sum_{l_{1} \cdots l_{m}} l_{i} n_{x}^{l_{1}, \cdots, l_{m}}}{\sum_{i=1}^{m}\left[n_{i} \exp \left(-\frac{W_{x}^{i}-k T \ln q_{x}^{i}+z_{x} W_{i}^{1}-z_{x} k T \ln q_{i}^{1}}{z_{x} k T}\right)\right]} \\
&= \frac{z_{x} n_{x}}{\sum_{i=1}^{m-1}\left[\exp \left(\beta_{i}-\frac{W_{x}^{i}-k T \ln q_{x}^{i}+z_{x} W_{i}^{1}-z_{x} k T \ln q_{i}^{1}}{z_{x} k T}\right)\right]} \\
& \times \frac{\left(1+\sum_{i=1}^{m-1} \exp \beta_{i}\right)}{\sum_{i=1}^{m} \sum_{l_{1} \cdots l_{m}} l_{i} n_{x}^{l_{1} \cdots l_{m}}=z_{x} n_{x} .}
\end{aligned}
$$

One gets from the last equality of eq. (41) and eq. (35) the following expression for the number of atoms of component $x$ in the system

$$
\frac{n_{x}^{*}}{\rho_{x} n_{s}-n_{x}^{*}}=\left\{\sum_{i=1}^{m}\left[\frac{n_{i}}{n_{s}} \exp \left(-\frac{W_{x}^{i}-k T \ln q_{x}^{i}+z_{x} W_{i}^{1}-z_{x} k T \ln q_{i}^{1}}{z_{x} k T}\right)\right]\right\}^{z_{x}} \exp \left(\frac{\mu_{x}}{N k T}\right) .
$$

To calculate the solubility of component $x$ in a multi-component system from the above equation, one requires a knowledge of the values of $W$ 's and $q$ 's. For this, one can take advantage of the thermodynamic properties of component $x$ in pure solvents corresponding to the components of the system. For the number of atoms of component $x$ in a solvent containing $n_{s}$ atoms of component $i$ only, one gets the following expression from eq. (43): 


$$
\begin{aligned}
\frac{n_{x}^{* i}}{\rho_{x} n_{s}-n_{x}^{* i}} & \\
= & \exp \left(-\frac{W_{x}^{i}-k T \ln q_{x}^{i}+z_{x} W_{i}^{1}-z_{x} k T \ln q_{i}^{1}}{k T}\right) \\
& \quad \times \exp \left(\frac{\mu_{x}}{N k T}\right) .
\end{aligned}
$$

Elimination of $W$ 's and $q$ 's from eq. (43) with the help of eq. (44) and similar expressions for the solubility of $x$ in other pure solvents, one gets the following general expression which correlates the solubility of component $x$ in a multi-component system with that in pure components :

$$
\begin{aligned}
Y_{x}^{(1,2, \cdots, m)=}= & {\left[\left(Y_{1}\left(Y_{x}^{(1)}\right)^{1 / z_{x}}+Y_{2}\left(Y_{x}^{(2)}\right)^{1 / z_{x}}+\right.\right.} \\
& \left.\cdots+Y_{m}\left(Y_{x}^{(m)}\right)^{1 / z_{x}}\right]^{z_{x}},
\end{aligned}
$$

where $Y_{i}, Y_{x}^{i}$ and $Y_{x}^{1,2, \cdots, m}$ are the lattice ratios of component $i, x$ in component $i$ and $x$ in multi-component system in equilibrium with a surrounding phase having $b_{x}$ as the chemical potential for component $x$, respectively. In the above expression $n_{x}$ is considered to be negligibly small as compared to the total available sites in the system. Lattice ratios in the above expression are defined ${ }^{(4)}$ as

$$
Y_{i}=\frac{n_{i}}{n_{s}}, \quad Y_{x}^{(i)}=\frac{n_{x}^{* i}}{n_{s}}, Y_{x}^{(1,2, \cdots, m)}=\frac{n_{x}^{*}}{n_{s}} .
$$

\section{Applications and Discussion}

Equation (45) is applicable only to the solution of those interstitial components which obey for their interaction energies and internal partition functions the linear relationships represented by eq. (4)-(7). In the case of a binary substitutional solvent eq. (45) leads to an expression which forms a special case (when $h=0$ ) of the following expression developed by Wagner ${ }^{(5)}$ :

$$
\begin{aligned}
Y_{x}= & \sum_{i=0}^{z_{x}}\left[{ }^{z_{x}} C_{i}\left\{Y_{A}\left(Y_{x}^{A}\right)^{1 / z_{x}}\right\}^{i}\left\{Y_{B}\left(Y_{x}^{B}\right)^{1 / z_{x}}\right\}^{\left(z_{x}-i\right)}\right. \\
& \left.\times \exp \left\{\frac{i\left(z_{i}-i\right) h}{2 R T}\right\}\right] .
\end{aligned}
$$

The above expression is derived under the assumption that the energy of an interstitial component is of the following parabolic form

$$
W_{x}^{l_{A}}=\frac{l_{A}}{z_{x}} W_{x}^{A}+\frac{z_{x}-l_{A}}{z_{x}} W_{x}^{B}+\frac{l_{A}\left(z_{x}-l_{A}\right)}{2 R T} h .
$$

Further Wagner ${ }^{(5)}$ has shown that the value of the system dependent constant $h$ is small in case of nitrogen whereas it is appreciable for oxygen in most of binary substitutional solvents. Block $^{(6)}$ has assumed that the bend energy of the pairs among the surrounding atoms is also affected by the presence of interstitial component. Similar assumptions have also been made by Alcock and Richardson ${ }^{(8)}$ and Jacob and Alcock ${ }^{(9)}$ in their treatments on the solubility of oxygen in binary and ternary solvents. Kapoor ${ }^{(10)}$ has shown that in binary solvent these effects in case of nitrogen are small as compared to the experimental errors in determining the thermodynamic data on nitrogen. Thus, it is expected that eq. (45) is applicable to the dilute solutions of nitrogen in multicomponent systems. Blossey and Phelke ${ }^{(11)}$ have studied the thermodynamics of dilute solution of nitrogen in ternary $\mathrm{Fe}-\mathrm{Co}-\mathrm{Ni}$ system at $1600^{\circ} \mathrm{C}$. They have determined the standard free energy of the reaction

$$
\frac{1}{2} N_{2}=N(1 \mathrm{wt} \%) \text {. }
$$

In Fig. 1 their results and the calculated ones using eq. (45) are plotted. Calculations are done according to the expression

$$
Y_{N}^{\mathrm{Fe}, \mathrm{Co}, \mathrm{Ni}}=\left(0.352 Y_{\mathrm{Fe}}+0.165 Y_{\mathrm{Co}}+0.195 Y_{\mathrm{Ni}}\right)^{6}
$$

as nitrogen is expected to occupy octahedral sites with a coordination number of 6 . The

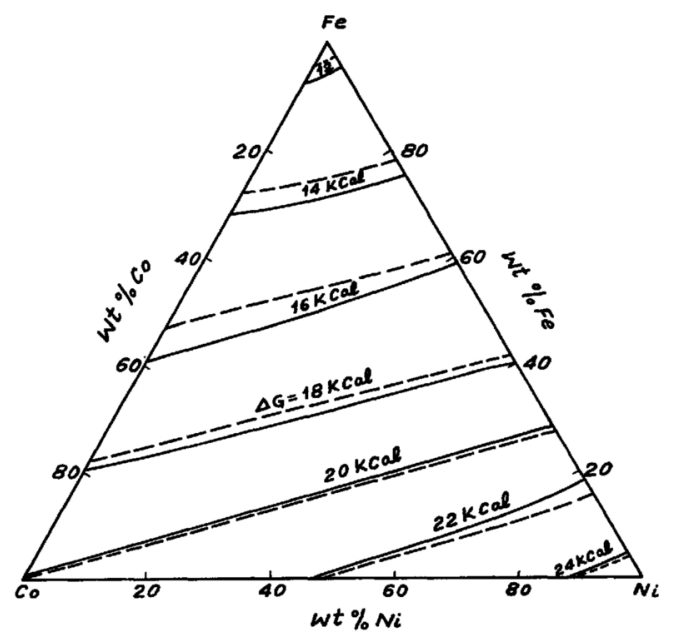

Fig. 1 Standard free energy of solution of nitrogen in liquid $\mathrm{Fe}-\mathrm{Ni}-\mathrm{Co}$ alloys at $1600^{\circ} \mathrm{C}$ and $1 \mathrm{~atm}$, nitrogen pressure. ...... experimental, _- calculated. 
coefficients of $Y_{\mathrm{Fe}}, Y_{\mathrm{Co}}$ and $Y_{\mathrm{Ni}}$ in the above expression are calculated from the free energy of reaction denoted by eq. (49) with pure molten metals as solvents. Figure 1 shows a satisfactory agreement between the experimental and the calculated values. As further examples for the application of eq. (45) standard free energy of the reaction represented by eq. (49) is calculated for systems $\mathrm{Fe}-\mathrm{Ni}-\mathrm{Cr}$ at $1600^{\circ} \mathrm{C}$. The calculation is done according to the expression

$$
Y_{N}^{\mathrm{Fe}, \mathrm{Cr}, \mathrm{Ni}}=\left(0.352 Y_{\mathrm{Fe}}+0.817 Y_{\mathrm{Cr}}+0.195 Y_{\mathrm{Ni}}\right)^{6},
$$

and the iso-free energy lines in this ternary system are shown in Fig. 2. The standard free energy of reaction (49) for pure molten chromium is taken as $-7.28 \mathrm{kcal} / \mathrm{g} \cdot$ atom of nitrogen and is derived from the works of Blossey and Phelke $^{(12)}$ and Hubert and Elliot ${ }^{(13)}$.

Another interstitial element of immense importance to metallurgy is carbon. But its solubility over the whole composition range has not yet been studied for any of the molten binary or ternary systems. However thermodynamic data on carbon is available for the low concentration ranges of a number of substitutional elements in iron base solvents. So, eq. (45) has to be modified accordingly to explain the thermodynamic behaviour of carbon in these systems. For this purpose, let us first

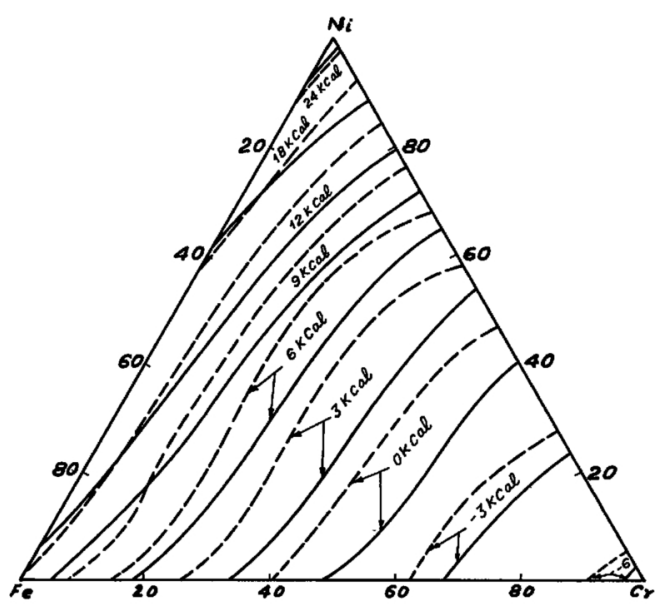

Fig. 2 Standard free energy of solution of nitrogen liquid $\mathrm{Fe}-\mathrm{Ni}-\mathrm{Cr}$ alloys at $1600^{\circ} \mathrm{C}$ and $1 \mathrm{~atm}$. nitrogen pressure. ......experimental, - calculated. consider a binary solvent of components $A$ and $B$. For this case one gets the following expression from eq. (45):

$$
\begin{gathered}
\ln Y_{C}^{A, B}=\ln Y_{C}^{A}+z_{x} \ln \left[Y_{A}+\left(\frac{Y_{C}^{B}}{Y_{C}^{A}}\right)^{1 / z_{x}} Y_{B}\right], \\
\varepsilon_{x}^{B}=-\left(\frac{\partial \ln Y_{C}^{A, B}}{\partial Y_{B}}\right)_{Y_{B} \rightarrow 0}, \\
\varepsilon_{c}^{B}=z_{x}\left[1-\left(\frac{Y_{C}^{B}}{Y_{C}^{A}}\right)^{1 / z_{x}}\right] .
\end{gathered}
$$

Elimination of $\left(Y_{C}^{B} / Y_{C}^{A}\right)^{1 / z}$ from eq. (52) with the help of eq. (54) leads to

$$
\ln Y_{C}^{A, B}=\ln Y_{C}^{A}+z_{x} \ln \left[1-\frac{\varepsilon_{c}^{B}}{z_{x}} Y_{B}\right]
$$

Similarly, for a component $i$ based multicomponent system, one can write the expression

$$
\ln Y_{C}^{1,2, \cdots, m}=\ln Y_{C}^{1}+z_{x} \ln \left[1-\sum_{i} \frac{\varepsilon_{c}^{i} Y_{i}}{z_{x}}\right] \text {. }
$$

For the case $\Sigma \varepsilon_{c}^{i} Y_{i} / z_{x} \ll 1$ the above expression yields

$$
\ln f_{c}^{1,2, \cdots, m} \equiv \ln \frac{Y_{C}^{1}}{Y_{C}^{1,2, \cdots, m}}=\sum_{i} \varepsilon_{c}^{i} Y_{i} .
$$

This expression has been shown to hold for the effect of low concentrations of $\mathrm{Cu}, \mathrm{Ni}, \mathrm{Co}, \mathrm{Mo}$, $\mathrm{Cr}, \mathrm{V}, \mathrm{Nb}$ on Henrian activity coefficient of carbon in liquid iron base alloys by Fuwa and Chipman ${ }^{(14)}$. But for higher concentrations of alloying element eq. (57) is expected to give better results.

As a concluding remark it can be stated that the present generalised approach based on statistical thermodynamics assuming linear dependence of energies of interstitial atoms on the surrounding substitutional ones holds good for nitrogen and carbon, but in the case of oxygen one has to consider the quadratic terms for the energies of the interstitial atoms.

\section{Acknowledgements}

The author is thankful to Mr. G. C. Kaushal for the help given to complete the work.

\section{Nomenclature}

$E_{i}, E_{\max }$ The energies of the $i$ th and most probable state of the system respectively. $E_{\text {sub }}, E_{\text {int }}$ Energy contributions due to sub- 
stitutional and interstitial atoms respectively.

$f_{c}^{1,2, \cdots, m}$ Henrian activity coefficient.

$g^{\left(E_{i}\right)}, g^{\left(E_{\max }\right)}$ Number of configurations of the states having a total energy of $E_{i}$ and $E_{\max }$ respectively.

$g_{\text {sub }}, g_{\text {int }}$ Number of configurations of substitutional and interstitial atoms in the states having $E_{\max }$ as the total energy.

$h\left(n_{s}, n_{x}^{*}\right)$ normalising factor.

$k$ Boltzmann's constant.

$N$ Avogadro's number.

$n_{1}, n_{2}, \ldots, n_{m}$ Number of atoms of components $1,2, \ldots, m$ respectively in the system.

$n_{x}^{i}, n_{x}^{i *}$ Number of atoms of component $x$ in the $i$ th and most probable state respectively of the system.

$n_{s}, n_{\text {int }}, n_{v}$ Total number of substitutional, interstitial and vacant interstitial sites in the system.

$n_{x}^{l_{1}, l_{2}, \cdots, l_{m}}$ Number of $x$ atoms having $l_{1}$, $l_{2}, \ldots, l_{m}$ atoms of components $1,2, \ldots$, $m$ respectively their first coordination shell. $n_{v}^{p_{1}, p_{2}, \cdots, p_{m}}$ Number of vacancies having $p_{1}$, $p_{2}, \ldots, p_{m}$ atoms of components 1 , $2, \ldots, m$ respectively in their first coordination shell.

$q_{x}^{i} \quad$ Internal partition function of an $x$ atom surrounded by only atoms of component $i$. $q_{x}^{l_{1}, \cdots, l_{m}}$ Internal partition function of an $x$ atom surrounded by $l_{1}, l_{2}, \ldots, l_{m}$ atoms of components $1,2, \ldots, m$ respectively.

$q_{k}^{j_{1}, \cdots, j_{m}, 1}$ Internal partition function of an atom of component $k$ surrounded by $j_{1}$, $j_{2}, \ldots, j_{m}$ atoms of components $1,2, \ldots$, $m$ respectively and one atom of component $x$.

$q_{k}^{j_{1}, \cdots, j_{m}, 0}$ Internal partition function of an atom of component $k$ surrounded by $j_{1}$, $j_{2}, \ldots, j_{m}$ an atom of components 1 , $2, \ldots, m$ respectively.

$W_{x}^{i} \quad$ Energy of an $x$ atom surrounded by atoms of component $i$.

$W_{x}^{l_{1}, \cdots, l_{m}}$ Energy of an $x$ atom surrounded by $l_{1}, l_{2}, \ldots, l_{m}$ atoms of components 1 , $2, \ldots, m$ respectively.

$W_{k}^{j_{1}, \cdots, j_{m}, 1}$ Energy of an atom of component $k$ surrounded by $j_{1}, j_{2}, \ldots, j_{m}$ atoms of components $1,2, \ldots, m$ respectively and an atom of component $x$.
$W_{k}^{j_{1}, \cdots, j_{m}, 0}$ Energy of an atom of component $k$ surrounded by $j_{1}, j_{2}, \ldots, j_{m}$ atoms of components $1,2, \ldots, m$ respectively.

$Y_{i}$ Lattice ratio of the substitutional component $i$.

$Y_{x}^{i}, Y_{x}^{1,2, \cdots, m}$ Lattice ratio of solute $x$ in pure solvent of component $i$ and in multicomponent solvent containing components $1,2, \ldots, m$ respectively when the solvent is in equilibrium with the surrounding phase having as the chemical potential for component $x$.

$z_{x}, z_{s x}$ Number of substitutional sites around an interstitial site and vice versa, respectively.

$\varepsilon_{c}^{i} \quad$ Interaction parameter.

$b_{x}$ Chemical potential of component $x$.

$\rho_{x}$ Number of interstitial sites per substitutional site.

$\Omega\left(n_{1}, \ldots, n_{m}, n_{x}\right)$ Configurational partition function of the system containing $n_{1}$, $n_{2}, \ldots, n_{m}, n_{x}$ atoms of components 1 , $2, \ldots, m$ and $x$ respectively.

$\Xi$ Semi-grand partition function of the system.

\section{REFERENCES}

(1) R. H. Fowler and E. A. Guggenheim: Statistical Thermodynamics, Cambridge University Press, (1960).

(2) I. Prigogine: The Molecular Theory of Solutions, North Holland Pub. Co., Amsterdam, (1957).

(3) B. Paseal, J. C. Mothiou, D. Desre and E. Bonniar: J. Chim. Phy., 68 (1971), p. 771, 782, 787.

(4) J. F. Elliot and J. Chipman: Proc. Int. Sym. on Met. Chem.-Applications in Ferrous Metallurgy, Sheffield, (1971), p. 348.

(5) C. Wagner: Acta Met., 21 (1973), 1297.

(6) U. Block: Met. Trans., 1 (1970), 2018.

(7) C. B. Alcock and F. D. Richardson: Acta Met., 6 (1958), 383.

(8) C. B. Alcock and F. D. Richardson: Acta Met., $8(1960), 883$.

(9) K. T. Jacob and C. B. Alcock: Acta Met., 20 (1972), 221.

(10) M. L. Kapoor: Int. Met. Reviews, to be published.

(11) R. G. Blossey and R. D. Phelke: Trans. Met. Soc. AIME, 236 (1966), 566.

(12) R. G. Blossey and R. D. Phelke: Trans. Met. Soc. AIME, 242 (1968), 2457.

(13) J. C. Kumbert and J. F. Elliot: Trans. Met. Soc. AIME, 218 (1960), 1076.

(14) T. Fuwa and J. Chipman: Trans. Met. Soc. AIME, 213 (1959), 708. 